\title{
A Rare Cause of Acute Abdomen - The Torsioned Intra abdominal Testicular Tumor
}

\author{
Henok seife ${ }^{1}$, Tadesse Habteyohhanes ${ }^{2}$ \\ ${ }^{1}$ General surgeon Addis Ababa university medical faculty \\ 2 General surgeon Debre-markos Referral Hospital
}

Correspondence to: Henok Seife, Email: henokyees@gmail.com

\section{Introduction}

Cryptorchidism is a developmental defect in which the testes fail to descend completely into the scrotum. Isolated cryptorchidism affects $3 \%$ of full-term male newborns. True undescended testis is unilateral in 80\% cases. Approximately 70 77\% of cryptorchid testes will spontaneously descend, usually by 3 months of age. Approximately $10 \%$ of testicular tumors arise from undescended testes. The higher the position of an undescended testis, the greater is the risk for development of malignancy. Almost half of the tumors that occur in testes are located abdominally, with 6-fold higher frequency than in an inguinal testis. The most common tumor that develops from the cryptorchid testis is the seminoma. Complications of intraabdominal testis such as torsion, rupture and bleeding are rare ${ }^{1 .}$

The risk of developing a germ cell tumor when a cryptorchid testis is intra-abdominal is about $5 \%$.In mixed groups of men treated for cryptorchidism, the risk is typically 3.6 to 7.4 times higher than in the general population. The incidence curve increases steeply after the onset of puberty, and occurrences are most frequent in men in their 20s and 30s; $50 \%$ are diagnosed before the age of 35 . While orchidopexy improves fertility, it does not alter the risk of developing carcinoma. Rather, it allows clinical surveillance of patients with a previously impalpable gonad. Ultrasonography, magnetic resonance imaging, computed tomography and Gallium scanning are usually used in the monitoring of an intra-abdominal testis ${ }^{1}$.

Acute abdomen and massive bleeding is a very rare presentation in cryptorchid testicular tumor with two cases of ruptured intra-abdominal seminoma reported in the literature ${ }^{1}$.

Intra-abdominal neoplastic testicular torsion is a very rare clinical condition, which is normally not considered during differential diagnosis of acute appendicitis. This is most often observed in patients after puberty, without any previous specific symptoms apart from the absence of the testis in the scrotum and inguinal canal or periodic pain in the right lower abdomen ${ }^{2}$. An increased diagnostic yield is dependent on an expedient and comprehensive preoperative evaluation. This consists of a detailed past surgical history, a thorough physical examination, and close inspection of the preoperative abdominal films ${ }^{3}$.

There are 36 reports of an intraabdominal testicular tumor presenting with torsion. The most common histologic types, in descending order, were seminoma, sarcoma, teratoma, embryonal carcinoma, and choriocarcinoma. Common presenting signs and symptoms included pain and/or tenderness, fever, nausea and/or vomiting, and a 
palpable mass. Most seminoma patients received adjuvant radiation therapy whereas one patient with choriocarcinoma received adjuvant chemotherapy ${ }^{4}$.

\section{Case Report -}

The patient was a 29 years old male patient who presented to menellik II hospital (one of the referral hospitals in Addis Ababa Ethiopia whose surgical department is affiliated by Addis Ababa university medical faculty department of surgery) emergency department at mid night having a referral from a health center with a presumed diagnosis of acute appendicitis after he presented with lower abdominal pain of 24 hours duration. The pain started in the left groin area and radiated to the right lower quadrant of the abdomen with a mass felt in the area. He had one episode of vomiting of ingested matter. He had such type of complaints two years back, then had milder symptoms and resolves by itself in $3-4$ days.. He had noticed an empty scrotum since 15 years back. Married three years back but no children yet and claims to use family planning

No other bowel or urinary complaint and no other medical illness. No fever, weight loss and anorexia or night sweating

At presentation, his BP was 110 / $70 \mathrm{mmHg}$ with pulse rate of $104 \mathrm{bpm}$ and respiratory rate of 20 per minutes and a temperature of $36.5^{\circ} \mathrm{C}$. There was no pallor or icterus noticed on examination of the eyes and mucous membranes of the mouth and no finding except for the abdominal findings Where it was found to be full with no movement on respiration and hypoactive bowel sounds, tympanytic at epigastric area and dull on the RLQ and supra umbilical area. Had tenderness and rigidity with guarding on the whole lower abdomen difficult to appreciate mass because of severe tenderness.

Digital rectal examination was non revealing with only scanty stool on examining finger. On Genitourinary system examination the scrotum was found to be empty on the left side and no testes in the left inguinal canal.. The right testis was in the scrotum. No other pertinent finding on physical examination.

With the provisional diagnosis of acute abdomen the patient was investigated with routine $\mathrm{CBC}$ and WBC was 12500 with $81 \%$ neutrophils and 9.2\% lymphocytes. Hgb was 12.9 , PLT was 212000 and urine analysis was without abnormal finding no further investigations were done as acute abdomen was assured and at midnight further investigation are not available.

With the same working diagnosis of acute abdomen secondary to complicated appendicitis to rule out complicated testicular mass he was prepared and taken to the operation room. When he was anesthetized on the operation table the mass in the abdomen became so obvious and gave a more likely impression of intra abdominal mass secondary to testicular mass. (Figures 1 and 2) 


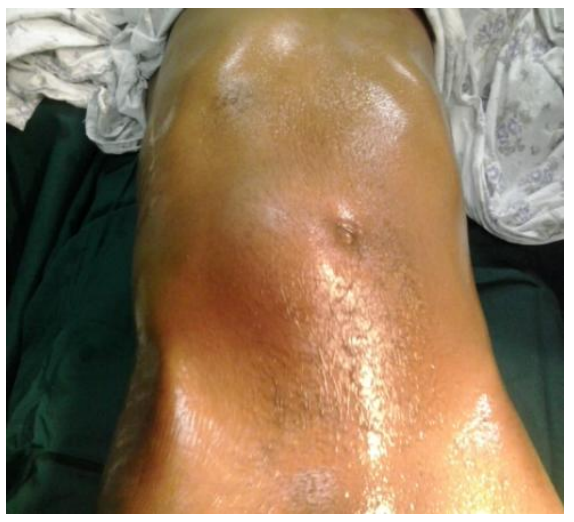

Figure 1.

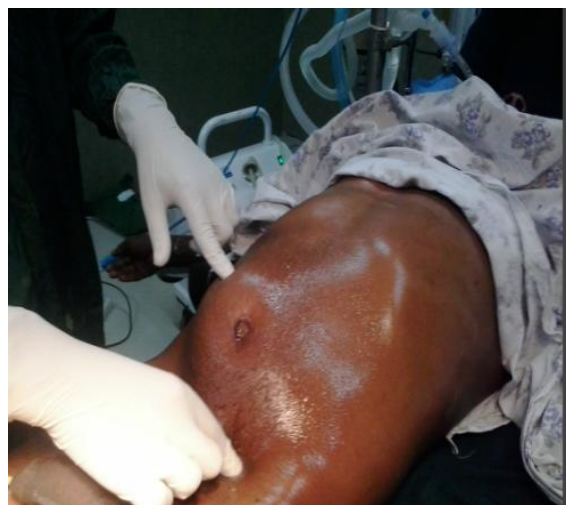

Figure 2

He was operated through a lower midline abdominal incision and the finding was: 360 degree clock wise twisted left tests $(15 \times 10 \mathrm{~cm})$ at its pedicle, Gangrenous, Highly vascularized mass. There was no attachment of the mass with the adjacent structures or no para aortic or other intra abdominal lymphadenopathy (Figures 3 and 4). What was Done was Mass excised area washed with normal saline and abdomen closed in layers.,

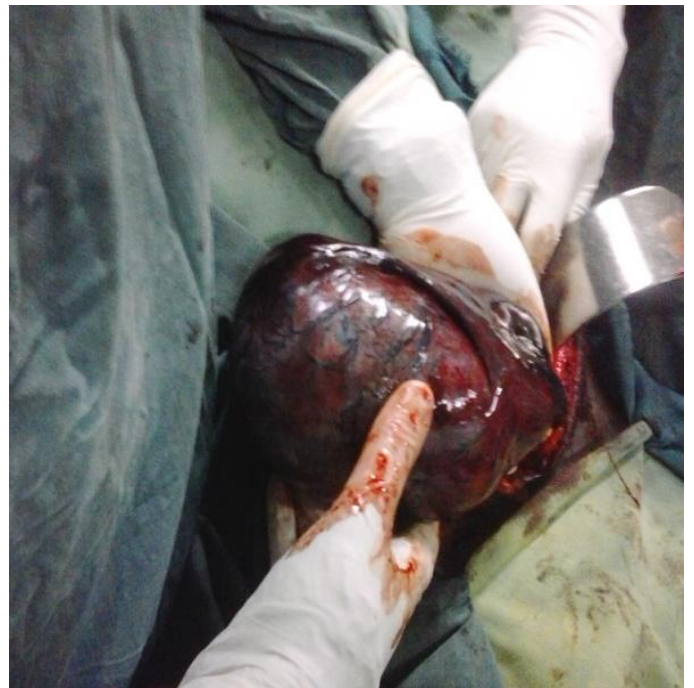

Figure 3

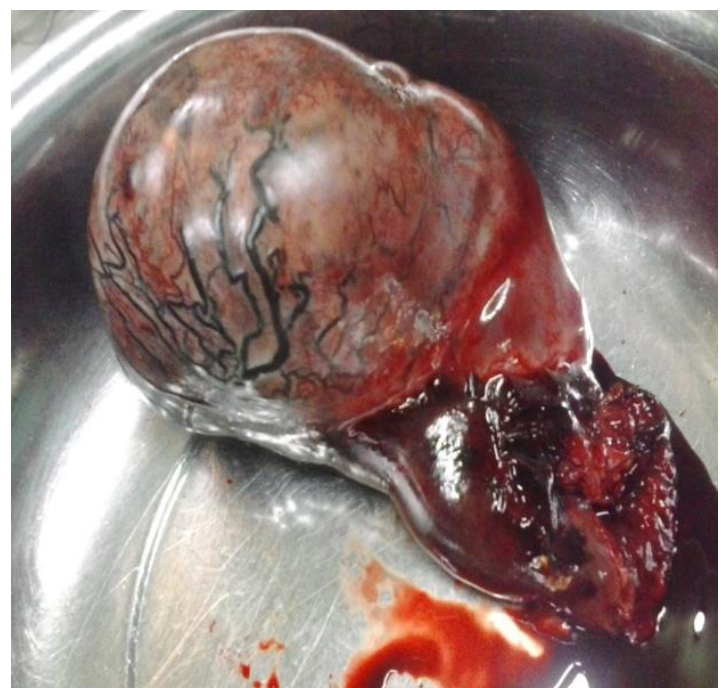

Figure 4.

The tissue subjected for histopathological examination. The patient was discharged after two days. The histopathology results revealed a testicular seminoma.and patient sent for onchology for further followup

\section{Discussion}

Testicular tumors are not uncommon but complicated intra abdominal testicular tumors with a torsion or rupture are rare findings. The diagnosis of such cases of acute abdomen with complicated intra abdominal testicular tumor complications preoperatively is usually difficult as they mimic one of the emergency conditions of acute abdomen like acute appendicitis or diverticulitis. As in this patient it could give some clues like absence of testes in the scrotum, longstanding history of lower 
abdominal pain or mass but it might not give any clue to the diagnosis as it is true for most such presentations. Making a preoperative diagnosis of complicated intra abdominal testicular tumors needs a high index of suspicion and especially the evaluation of the scrotum is very important to consider the condition. The outcome of treatment is usually good in almost all published cases and also in our case. Patients are unlikely to have postoperative complication even if the diagnosis is made intra operatively. Patients need further follow-up and adjuvant chemotherapy as is indicated by the histology of diagnosis.

\section{References}

1. N Kumar, B Rehmani. Spontaneous Rupture Of An Intra-Abdominal Testicular Tumor: A Case Report. The Internet Journal of Surgery. 2012 Volume 28 Number 3.

2. Tomasz Staniuk $\underline{1}$ / Łukasz Szymański르 / Mirosław Trocha른-Torsioned Intraabdominal Testicular Tumor Diagnosed during Surgery Performed due to Suspicion of Acute Appendicitis Polish Journal of Surgery. Volume 84, Issue 10, Pages 526-530, ISSN (Online) , ISSN (Print) 0032-373X, DOI: 10.2478/v10035-012-0088-y, December 2012

3. Ronald G. Frank M.D., Perry S. Gerard, Jude T. Barbera,Kris Lindsay, Gilbert J. Wise Torsion of an intraabdominal testis tumor presenting as an acute abdomen, Urologic radiology December 1990, Volume 12, Issue 1, pp 50-52

4. Khorasani R, Capelouto CC, Moore FD Ir Silverman SG Loughlin KR Torsion of intraabdominal testicular tumors. A case report.. ${ }^{1}$ Department of Urology, Brigham and Women's Hospital, Harvard Medical School, Boston, Massachusetts, 02115, USA.।

5. Tomasz Staniuk쓰 / Łukasz Szymański² / Mirosław Trocha :-Torsed Intraabdominal Testicular Tumor Diagnosed during Surgery Performed due to Suspicion of Acute Appendicitis - Polish Journal of Surgery. Volume 84, Issue 10, Pages 526-530, ISSN (Online) , ISSN (Print) 0032-373X, DOI: 10.2478/v10035-012-0088-y, December 2012 